\title{
Integration of a QoS aware end user network within the TISPAN NGN solutions
}

\author{
I. Vidal, J. Garcia, F. Valera, I. Soto, A. Azcorra \\ University Carlos III of Madrid, Telematic Engineering Department \\ \{ividal, jgr, fvalera, isoto, azcorra\}@it.uc3m.es
}

\begin{abstract}
The development of specifications for Next Generation Networks has introduced a new network paradigm that has features and capabilities to ensure the provisioning of new value-added multimedia services over the broadband access technologies that are currently being deployed in the market. In this respect, the TISPAN group from ETSI is working in the specification of a Next Generation Network, mainly based on IMS as the service signalling architecture. Nevertheless, the first release of the TISPAN NGN does not consider the QoS provisioning mechanisms in the end user environment. QoS support will necessarily require to extend the QoS scope to the client premises, in order to provide a real end-to-end QoS. In this scenario, the figure of a Residential Gateway in the user network plays a crucial role. In this paper, an architecture is presented to allow the automatic configuration of the QoS parameters in a Residential Gateway directly connected to a TISPAN compliant Next Generation Network. This architecture should support the processing of the SIP signalling flows exchanged between the end user environment and the Core IMS in the $N G N$, deriving relevant information from the SDP payloads and accordingly configuring the QoS parameters in the user network environment.
\end{abstract}

\section{Introduction}

The idea of a Next Generation Network (NGN) is commonly understood as a new kind of network architecture together with its related technologies. An NGN architecture is developed with the purpose of integrating different multiple services (data, voice, video, etc.) and of facilitating the convergence of fixed and mobile networks.

Based on the definition provided by the ITU-T [5], these NGNs are usually built on top of a switched packet technology (like the Internet Protocol) and are capable of providing Quality of Service on the transport layer while keeping it independent from the service layer and with the provisioning of generalized mobility of users and services.
A core component in NGN architectures is the one defined by the 3GPP, the IP Multimedia Subsystem (IMS), that was introduced in the release 5 of 3GPP standards in 2002 as an IP-based architecture used to signal the new value-added services with QoS requirements envisioned for UMTS.

Although IMS is conceptually designed to be independent from the technology used in the access network, the standards developed by the $3 \mathrm{GPP}$ from release 5 are mainly focused on the UMTS IP connectivity access network (IPCAN). In this respect, in 2004, ETSI and 3GPP started to cooperate in the ETSI TISPAN group, trying to use IMS over other access networks different from the 3GPP IPCAN. As a result of this ongoing work, the first release for TISPAN Next Generation Network (NGN) was published at the beginning of 2006.

In this article the QoS model defined in TISPAN NGN is described, analysed and extensions are proposed to achieve some synergy between this model and a typical broadband access network architecture of the near future. In particular this article uses as access network architecture the one defined by the MUSE European research project. MUSE [7] is a large integrated $R \& D$ project on broadband access that aims at a consensus view of the future access and edge network, achieved by the co-operative research of almost all major players in Europe (36 partners, including system and component vendors, telecom operators, SMEs and universities and research institutes).

In MUSE, the provisioning of an end-to-end QoS environment is an important issue but the model defined by TISPAN does not reach the home network (end-user network). This article proposes the extension of the NGN model beyond the access node, so that it is possible for the users to receive services with assured quality down to their own terminals.

The rest of the article is structured as follows. The second section is focused on an overview of the QoS mechanisms provided by TISPAN for the core, the access and the end user networks. The third section covers the QoS solutions proposed by the MUSE European research project in the home network, focused on a key element, the Residen- 


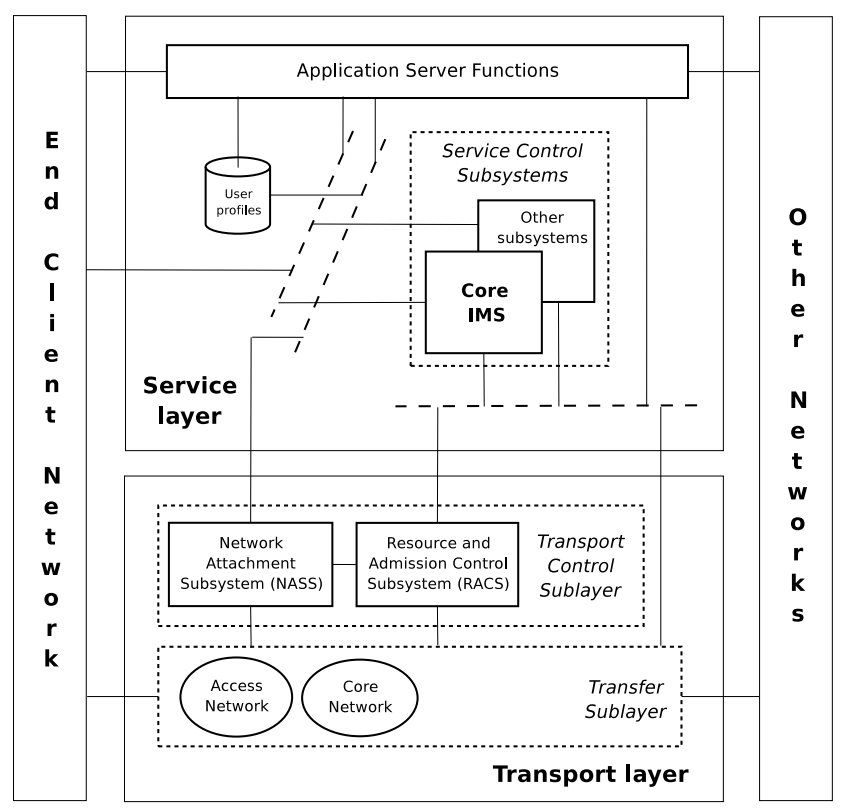

Figure 1. TISPAN NGN architecture overview

tial Gateway (RGW). The fourth section describes an alternative in order to support end to end QoS based on TISPAN concepts, integrating the end user network by means of the RGW. The final section describes the most important conclusions achieved along the article.

\section{QoS provisioning in TISPAN solutions}

The main objective of TISPAN Release 1 is to provide an extensible platform for the development of future services and architecture for the NGN (see [15]). TISPAN NGN will support a variety of user equipment, from simple legacy telephones to client networks connected through a residential gateway.

Currently, Release 2 is under development and is scheduled to be published in 2007, so this paper will focus on the specifications contained in the Release 1 . In the next subsection a brief description about the TISPAN NGN Release 1 functional architecture is given, to provide the reader with a general view about the main entities that compose the TISPAN NGN. Out of these, in Sec. 2.2 the paper will focus on the functional entities that are related with QoS provision in the access and core networks.

\subsection{TISPAN NGN architecture}

The functional architecture of TISPAN NGN in Release 1 is described in detail in [10]. Figure 1 covers a simplified overview of this architecture.

As it is shown in this figure, the functional architecture is structured in two layers, the transport layer and the service layer. Both layers are built up by a set of functional entities and subsystems and each subsystem is in turn defined as sets of new functional entities, interacting between them to provide the functionality envisioned for the subsystem.

Transport layer. The transport layer provides IP connectivity to the user equipment in client premises. The functionality supported by this layer is divided in two sublayers: a transport control sublayer and a transfer sublayer.

The transport control sublayer comprises the following subsystems:

- The Resource and Admission Control Subsystem (RACS) performs policy control, resource reservation and admission control functions in the NGN. The RACS subsystem provides applications with the capability of reserving resources from the transport networks, guaranteeing QoS provision for the valueadded services in the NGN. Section 2.2 briefly covers the RACS architecture and further details can be found in [11].

- The Network Attachment Subsystem (NASS) provides user equipment initialisation so as to access to NGN services, network level authentication, network access authorisation, access network configuration and location management. Further details about the NASS can be found in [12].

Finally, the functional entities that constitute the transfer sublayer are covered in detail in [10] and those that are relevant in the QoS provisioning processes will be briefly reviewed in Sect. 2.2.

Service layer. The service layer comprises a set of subsystems that provide service control functionalities. The following service control subsystems have been proposed in TISPAN NGN Release 1: the Core IMS, the PSTN/ISDN Emulation Subsystem, the Streaming Subsystem and the Content Broadcasting Subsystem. Out of these, the Core IMS [13] provides the means to negotiate SIP-based multimedia services to NGN terminals. It is a subset of the IMS as it was defined in the 3GPP Release 6 specifications, restricted to the session control functionalities.

The service layer also provides a set of common components [10]. One of these components is the Application Server Function (ASF). The functionality of the ASF in the NGN architecture consists of providing value-added services to the NGN terminals. These can be standalone services or can be provided on top of an established session, for example an IMS session. In the former case, the ASF may directly interact with the RACS subsystem for QoS provision; in the last case, the ASF relies on the service control 


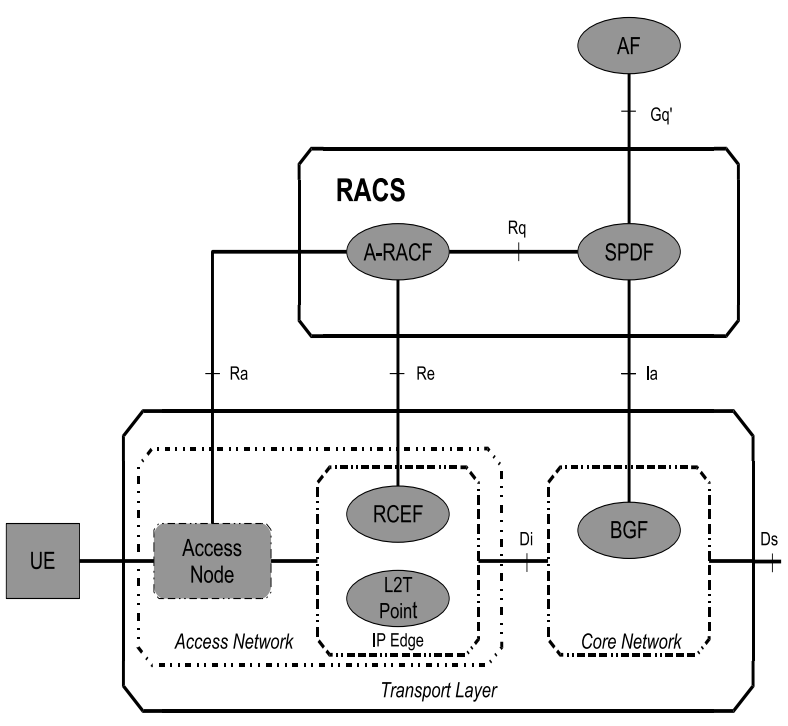

Figure 2. RACS functional architecture

subsystem that provides the session, which in turn interacts with the RACS.

\subsection{Access and Core Network QoS solu- tions}

In this section a brief revision is provided about the QoS solutions that have been considered in the Release 1 of the TISPAN NGN. In this respect, the RACS subsystem plays a crucial role, implementing the resource reservation mechanisms from the transport networks that are essential to provide QoS support to applications and services in the NGN. These mechanisms are available both for ASF and for the service control subsystems in the service layer.

In Release 1 of the TISPAN NGN the RACS scope is limited to the access network, to the interconnection points between the access and core networks and to the interconnection points between core networks. As it is specified in [11] this first release does not require the RACS subsystem to cover the Core Network itself or the end client network, so that the standardization efforts are mainly focused on the access network, which is viewed as the most critical transport segment in respect to the QoS provision problem. Figure 2 shows an overview of the functional architecture of the RACS subsystem.

The Application Function (AF) interacts with the RACS subsystem to request QoS provisioning to services. This function is implemented in some functional entities from the service layer, such as the ASF and the P-CSCF of the Core IMS (see next subsection). The AF converts the QoS demands specified for the services to QoS information which is suitable for the RACS subsystem, and sends requests with this information to the SPDF through the Gq' interface [16].

The Service Policy Decision Function (SPDF) authorises each request, checking the information contained on it against the local policy established for the requester AF. If the request is successfully authorised, the SPDF determines if it must contact the A-RACF and/or the BGF to serve the received request.

The Access-Resource and Admission Control Function (A-RACF) provides the functionality for admission control and resource reservation over the access network to the SPDF. The A-RACF can accept or reject the requests received from the SPDF based on the admission control mechanisms. The interface between the SPDF and the A$\mathrm{RACF}$ is the Rq reference point [14]. The interface $\mathrm{Ra}$ is not standardized in Release 1.

The Resource Control Enforcement Function (RCEF) is located in the IP edge node of the access network, and perform the enforcement of the traffic policies that guarantee the resource reservation in this network segment. These traffic policies are specified by the RACS over the Re interface, and are based on opening/closing gates functions, packet marking and policing of upstream/downstream traffic. The Re reference point is not standardized in Release 1 .

Finally, the Border Gateway Function (BGF) provides the interface between two IP domains. The BGF provides policy enforcement and NAT functions that are under the control of the SPDF over the Ia reference point [11].

The RACS subsystem supports several schemes for resource management, two of which are indicated below:

- A reserve-commit resource management scheme, where the resources are reserved in a first request and are finally made available after a second commit request.

- A single-stage resource management scheme, where the commit procedure is implicit to the reservation request.

In addition, the RACS subsystem supports two different models for QoS control over the transport network:

- Guaranteed QoS. In this model the QoS is assured with absolute bounds on some or all of its parameters, like throughput or jitter. Guaranteed QoS is configured on the access network with the application of techniques such as throughput control and traffic policing in the IP edge node.

- Relative QoS. In this model the QoS is provided by class based differentiation. This QoS differentiation 
is configured in the IP edge node of the access network, where functionalities like packet marking are provided.

As a conclusion for this subsection it has been seen that Release 1 of TISPAN NGN does not consider QoS provision mechanisms in the end user environment. Nevertheless, extending the QoS scope to the customer premises will be essential to provide end to end QoS. In this respect, Sect. 3 briefly covers the QoS solutions that have been proposed in MUSE project for the home network, focusing on the fundamental role played by the RGW in this scenario.

\subsection{Interaction between the Core IMS and the RACS subsystem}

The session establishment procedures in IMS are based on the Session Initiation Protocol (SIP) [9] and the Offer/Answer model [8] of the Session Description Protocol (SDP) [4]. SDP provides the means to describe multimedia sessions, such as the IP addresses, ports, type of media streams, codecs or bandwidth requirements for the IP flows that will be transferred during the session, whereas the Offer/Answer model of SDP allows the session participants to reach an agreement on the parameters that describe the session that is being negotiated.

On the other hand, in the IMS architecture the ProxyCall Session Control Function (P-CSCF) is the first contact point for the user terminals in the system. All the SIP signalling messages that are sent from or received by any terminal must go via a P-CSCF. In TISPAN NGN the P$\mathrm{CSCF}$ in the Core IMS implements an AF function, interacting with the RACS subsystem to do the corresponding resource reservation for the services negotiated between the session participants.

As it is explained in [16] the P-CSCF will send service information to the RACS subsystem, in concrete to the SPDF, upon receiving any SIP message containing an SDP answer payload. This service information will be derived from the SDP answer and its corresponding SDP offer. Jointly, both SDP payloads describe completely the session as it has been negotiated up to that moment. Annex B in document [16] contains the SDP to service information mapping process that must be performed by the P-CSCF. In addition, the P-CSCF can request from the RACS subsystem a reserve-commit or a single-stage resource management scheme in the QoS reservation request.

\section{Extension of QoS provisioning towards the end user networks}

\subsection{QoS in an multiservice access environ- ment}

Up to date, many initiatives about Next Generation Networks have been proposed. The MUSE (Multi Service Access Everywhere) project [7] is one of these initiatives, and its main objective is the research and development of a future, low cost, multi-service access network, capable to deliver the next generation broadband services to every European citizen. MUSE ongoing work is divided in several subprojects where different technologies are considered and where 36 partners collaborate.

With the previous considerations, the Residential Gateway (RGW) is part of the user network, fitting at the boundary between home network and the access network. In MUSE, the RGW is viewed as an important block to extend the QoS to the home network so as to be able to provide end to end QoS to the services delivered to the end users. In this respect, the organization of the project includes a specific steering Task Force dedicated to the figure of the Residential Gateway.

\subsection{RGW QoS architecture}

Figure 3 shows the architecture for the RGW that has been designed and implemented in MUSE within the framework of high speed access networks (it has been recently demonstrated in [1]).

As it can be seen in this figure, this architecture has been mapped into two implementation layers. The application layer is mainly based on flexible Java components capable of managing the lower layers and capable of treating different signalling and application level protocols. The data layer, based on the Click! modular router platform [6], provides all the specific data switching functionality (further details on the implementation can be found in [3]).

Application layer. These blocks are implemented in a high level language, being Java the selected one in the implementation scheme.

In this way, it is easy to develop new applications in this level in order to be able to manage the specific operation of certain protocols. One of the key components in this level is the Click! Signalling Dispatcher (CSD). This element may be used to configure the data layer (Click! modular router) to extract or copy certain frames from the transport network and to redirect them to some specific applications.

For instance, in the case of TISPAN NGN, a new application can be designed and implemented to be able to process the SIP signalling messages that are exchanged 


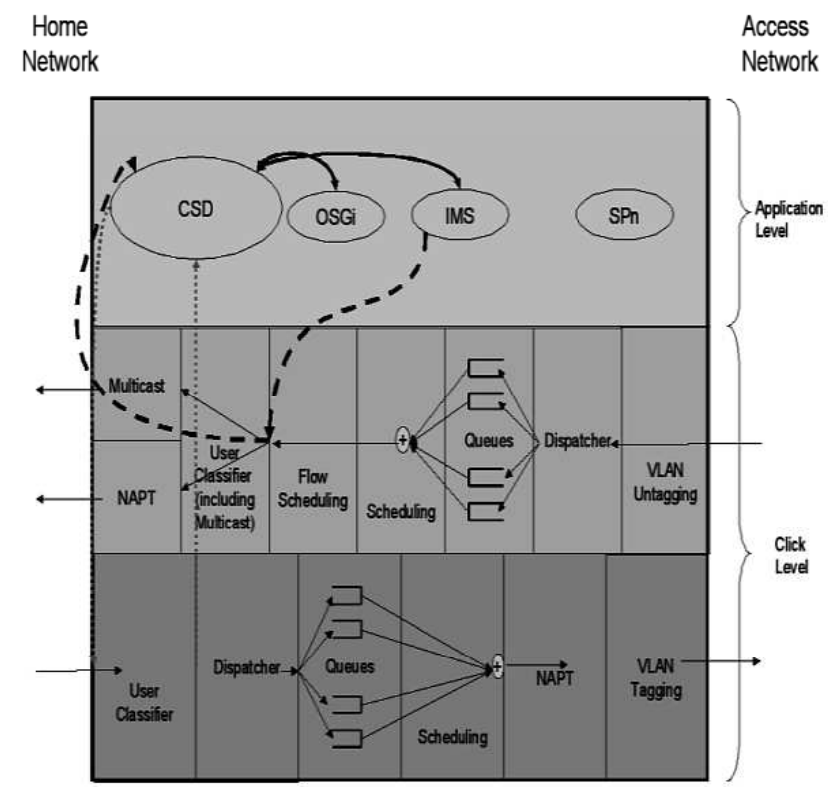

Figure 3. RGW implementation scheme

during the service negotiation between the terminals in the home network and the Core IMS in the NGN Core Network. In this case, the CSD would configure the data layer to redirect all the SIP messages to this application. These applications that are developed at this level are called Signalling Processes (SP).

Data layer. In order to design a flexible architecture at the data level, different types of blocks were defined to perform specific tasks. Following the scheme shown in Fig. 3, some of these blocks are detailed here for each traffic direction:

- Upstream:

- User Classifier: the administrator can specify classification rules to allow or deny certain flows. For example, a new rule could be added to classify all packets coming from a user IP phone as a high priority flow.

- Dispatcher: using the result of the previous classifier, this block pushes the frame towards the proper queue.

- Queues: this is a configurable block that allows an administrator to request a certain number of queues, fixing the size of each queue in terms of stored packets.

- Scheduling: this is the algorithm used to extract the packets from the queues.
- VLAN tagging: based on the result of the User Classifier, this block introduces a 802.1p VLAN tag in the Ethernet frame.

- Downstream: (User Classifier, Dispatcher, Queues and Scheduling have the same functionality as in the upstream direction):

- VLAN untagging: the VLAN tag is extracted from the Ethernet frame and an internal mark is attached to the packet (as meta-information).

- Flow scheduling: this classifier is used by the CSD to add or remove rules to extract or copy frames. Whenever a new application is registered in the application level, the CSD modifies this block in the Click! level to add a rule.

So, the RGW implements QoS provision mechanisms based on packet marking functions. In the direction of upstream traffic the different data flows that pass through the RGW are classified and placed into different queues. The extraction of the packets from the queues is scheduled to satisfy the constraints imposed by the QoS configuration and the extracted frames are tagged with 802.1p VLAN tags before being sent to the access network. A similar mechanism is employed in the downstream direction.

\section{Automatic QoS configuration in a TISPAN aware RGW}

As it has been explained in Sect. 2, the specifications of TISPAN NGN Release 1 are mainly focused on the QoS provision in the access network. Nevertheless, a complete service architecture with QoS support will necessarily require to extend to QoS scope to the customer premises, to provide end-to-end QoS. On the other hand, the RGW architecture presented in the previous section for the MUSE framework provides QoS capabilities in the end user environment.

Combining both approaches, in this section a new architecture for the presented RGW that supports automatic QoS management in a home network connected to a NGN compliant with the TISPAN NGN Release 1 is proposed. This architecture will process in the RGW all the SIP signalling messages exchanged between the end user network and the Core IMS, deriving from the SDP payloads the QoS demands for the session and accordingly configuring the corresponding QoS parameters in the home network.

\subsection{RGW architecture}

The new architecture, proposed for the RGW, is based on Signalling Processes (SP) and is in line with the RACS 


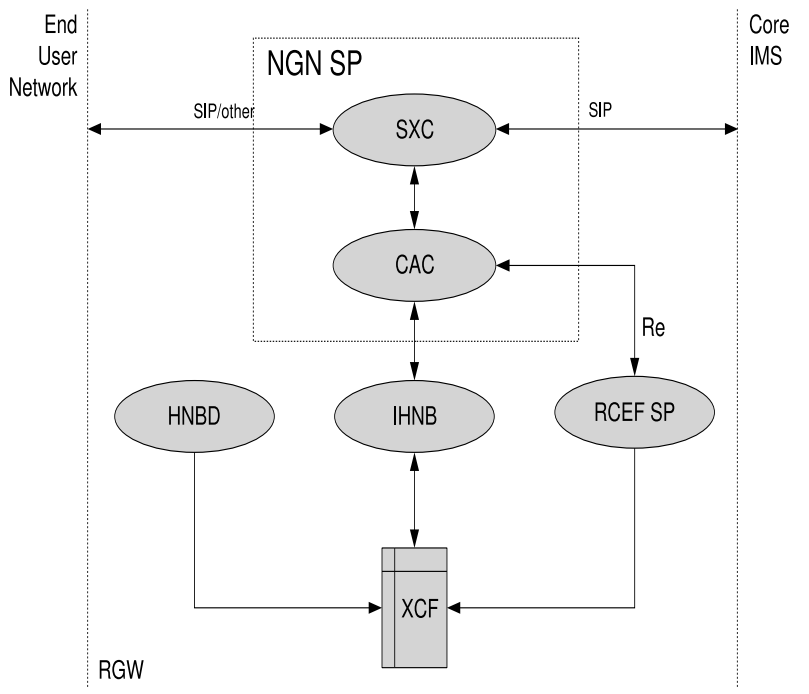

\section{Figure 4. Functional architecture in the RGW for automatic QoS configuration}

architecture as it has been explained in Sect. 2. Figure 4 shows a detailed overview of this functional architecture.

The NGN Signalling Process (NGN SP) is the responsible process for the management of the SIP signalling messages that are used to negotiate services between end users in the TISPAN NGN. It will process all the SIP signalling messages exchanged between the end user network and the Core IMS in the NGN Core Network. In a previous work [2] this model was validated to assure that the RGW could handle all these messages without a perceptible performance degradation. The NGN SP will include the following functional entities:

- SXC (Signalling Cross Connector). This entity will provide the following functionality:

- AF functionality: after receiving every SIP message containing a SDP answer, it will derive the corresponding service information from the SDP offer and the SDP answer as it is specified in [16]. With this information, the SXC can request the Call Admission Control (CAC) module to perform the QoS reservation mechanisms in the home network. In addition, if a reservecommit resource management scheme is used, once the IMS session is established it will contact the CAC to start the commit process for the reserved resources.

- SIP signalling proxy: this functionality will be necessary when the customer terminal in the home network is not TISPAN NGN compliant.
In this scenario, the SXC will behave as a signalling proxy on behalf of the legacy terminals, by generating the SIP signalling associated with the upstream and the downstream traffic and by exchanging this signalling information with the Core IMS. In addition, the SXC will also process each pair SDP offer/answer to do the corresponding resource reservation in the home network.

- CAC (Call Admission Control): with the service information provided by the SXC, the CAC will perform admission control functions, verifying if the QoS demands can be satisfied in the home network with the free resources. The information about the free resources will be provided to the CAC by the Instantaneous Home Network Bandwidth (IHNB) module. If the admission control process states that the requested QoS can be provided, then the CAC will contact with the RCEF SP to install new traffic policies capable of guaranteeing the resource reservation in the home network, and will indicate to the IHNB the set of resources that were reserved.

The CAC will support the reserve-commit and the single-stage resource management schemes. In respect to the QoS control model, the CAC will provide a relative QoS provision mechanism based on packet marking, which is the model actually implemented in the RGW.

The XML Configuration File (XCF) is an XML file parsed in memory that contains all the parameters that are necessary to configure the Click! router, such us the IP filters or the rules that specify which information that arrives to the RGW is sent to each signalling process. This file will also be used to save information about the available resources in the RGW and about the resources already reserved for IMS sessions.

The Home Network Bandwidth Detection (HNBD) detects, at start-up, the parameters that define the hardware resources that the RGW has available. This information will be stored in the $\mathrm{XCF}$, so it will be available to other functional entities.

The Instantaneous Home Network Bandwidth (IHNB) acts as intermediary between the $\mathrm{CAC}$ and the $\mathrm{XCF}$, hiding to the $\mathrm{CAC}$ the details about the internal configuration of the Click! router. The IHNB implements two functionalities on behalf of the CAC: first, it provides the CAC with information about the free resources (e.g. the free bandwidth to transmit over an interface), obtained from the information stored in the XCF. On the other hand, once the CAC notifies the IHNB that a concrete reservation has been done, it updates the information about reserved resources in the $\mathrm{XCF}$. 
The RCEF Signalling Process (RCEF SP) will implement some of the functionalities specified for a Resource Control Enforcement Function in [11], i.e. opening/closing gates and packet marking. The RCEF will apply the traffic policies established by the CAC of the NGN SP, by modifying the configuration of the Click! router, and guaranteeing the reservation of resources in the RGW.

Finally, the RCEF will provide an interface fully compatible with the Re interface defined in the RACS architecture detailed in [11]. This way, a new mechanism is provided that would allow a future scenario where the RACS subsystem in the NGN access network might directly access to the RCEF function in the RGW to manage the QoS policies in the client premises.

\subsection{Session establishment and resource management in the RGW}

Figure 5 shows the SIP signalling flow exchanged between a terminal, in the end user network, and the Core IMS to establish an IMS session for a VoIP call. The figure focuses on the processes that are performed in the RGW in respect to the automatic QoS management. In this example, a reserve-commit resource management scheme has been considered.

The procedure is as follows:

1-3. The terminal sends a SIP INVITE request from the end user network, including a SDP offer, to the PCSCF in the Core IMS. The RGW captures this message and sends it to the SXC. The SXC stores the SDP offer and forwards the SIP INVITE to the Core IMS. The request is processed in the Core IMS and forwarded to the IMS domain of the destination user.

4-5. The destination terminal answers back with a Session Progress message that contains an SDP answer. This message is received in the Core IMS that processes it and sends it to the terminal in the end user network.

6-9. The RGW captures the Session Progress message and sends it to the SXC. The SXC stores the SDP answer, derives the corresponding service information from the SDP offer and answer and sends a resource reservation request to the $\mathrm{CAC}$. The $\mathrm{CAC}$ performs the admission control procedures for the request. If the QoS demands can be satisfied with the free resources, then it does the corresponding resource reservation and contacts with the RCEF SP to install the traffic policies in the RGW that guarantee the QoS provision for the session. These traffic policies are based on packet marking functions. Finally, the CAC informs the SXC about the outcome of the process (if the requested QoS

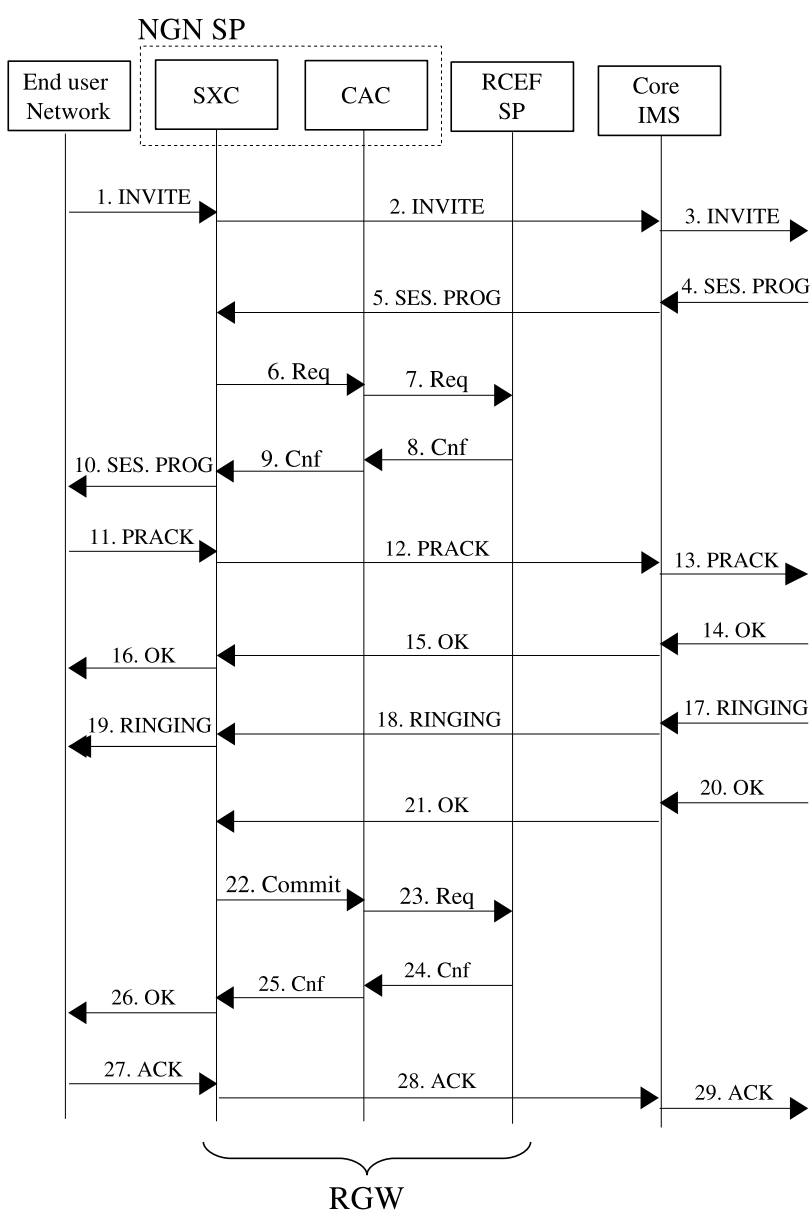

Figure 5. IMS session establishment and automatic QoS reservation in the RGW 
can not be granted, the SXC should finish the session establishment, exchanging the proper SIP signalling messages).

10. The SXC forwards the Session Progress message to the terminal in the end user network.

11. The terminal decides the final SDP parameters for the session and confirms the reception of the Session Progress with a PRACK message. This message may also contain an SDP payload that may be the same as the one that was received in the previous message or a subset. The terminal can also include a new SDP offer in this message, or even later using SIP UPDATE messages. Anyway, each SDP offer/answer exchange will cause a new interaction between the SXC and the $\mathrm{CAC}$ in the RGW.

12-13. The SXC receives the PRACK message and forwards it to the P-CSCF in the Core IMS. The Core IMS processes the message and sends it to the Core IMS of the destination terminal.

14-16. The destination terminal acknowledges the PRACK with an OK message. If the PRACK message included an SDP offer, the OK message will also contain a SDP answer. If the session description has changed, a new interaction between the SXC and the CAC must be performed as it has been specified in 6-9.

17-19. The destination terminal alerts its user about the incoming call and sends a SIP RINGING message to the originator terminal.

20-21. When the destination user answers the phone, the destination terminal sends a SIP OK message to the originator terminal, that finally arrives to the RGW. The RGW sends the message to the SXC.

22-25. The SXC sends a Commit request to the CAC, indicating the service information corresponding to the latest SDP offer and answer. The CAC commits the reserved resources, by opening the "gates" in the transport layer for the IP flows that are going to be transmitted in the session. Finally, the CAC reports to the SXC that the commitment has been performed.

26. The SXC forwards the SIP OK message to the terminal in the end user network.

27-29. The SIP OK message arrives to the originator terminal, and the user can start sending media. The terminal responds the SIP OK message with an ACK message, which arrives to the SXC and is forwarded to the Core IMS, where it is processed and finally sent to the Core IMS of the destination terminal.
As it can be seen, with a reserve-commit resource management scheme early traffic is forbidden over the transport layer during the session establishment, as the "gates" in the RGW are opened once the session is set up with the 200 OK message. Nevertheless, if the operator policy allows early traffic during the session set-up, a single-stage resource management scheme can be used. In this case the gates are opened in the reservation request (steps 6-9), so once the request is processed traffic can flow through the RGW.

\section{Conclusions}

The Release 1 of specifications for TISPAN NGN provides a framework that allows the introduction of next generation services with QoS requirements. But in this release, the scope of the QoS is mainly focused on the access network, and it is not considered any QoS provision mechanism for the end user network or for the Core Network itself. Nevertheless, whereas in the Core Network it can be assumed that this problem may be solved by other means (e.g. over provisioning), providing QoS support in the home network presents more critical problems.

In this scenario, the MUSE approach remarks the fundamental role of the RGW in the home network environment. The RGW fits between the customer equipment and the access network, and may be used to configure the local QoS parameters in the client premises.

In this paper, a new architecture is proposed in order to allow the automatic QoS configuration in a RGW that is connected to a TISPAN NGN compliant network, as it is specified by the documents in the Release 1 . This architecture is in line with the specification of the Resource and Admission Control Subsystem (RACS) of this release: it provides the processing of the SIP signalling flows that are exchanged between the home network environment and the Core IMS in the NGN Core Network, deriving from the SDP payloads all the information that defines the QoS demands for the sessions. With this information the admission control procedures in the home network are executed, including the possible installation of traffic policies in the RGW that guarantee the resource reservation in the end user network. The operation of the RGW in respect to QoS provisioning is transparent to the NGN, where the RACS subsystem provides the QoS management functions in the access network, in the edge between the access and core networks and between core networks.

But, although this architecture would allow to extend the QoS scope to the end user environment, additional issues must be studied further. The operation of the NGN SP in respect to the attachment of legacy terminals in the end user network, which are not compliant with the TISPAN NGN, must be considered in more detail. Also, more effort must 
be performed in the specification of the admission control mechanisms in the NGN SP, to make the adequate QoS enforcement decisions taking into account the free resources in the end user environment.

\section{Acknowledgements}

This article has been partially granted by the European Commission through the MUSE project (IST-026442), and the Spanish MEC through the CAPITAL project (TEC200405622-C04-03/TCM).

\section{References}

[1] F. Valera and J. Garcia and C. Guerrero and V. M. Ribeiro and V. Pinto. Demo of triple play services with qos in a broadband access residential gateway. In IEEE Infocom, April 2006. Barcelona, Spain.

[2] C. Guerrero, J. Garcia-Reinoso, F. Valera, and A. Azcorra. Qos management in fixed broadband residential gateways. LNCS, 3754:338-349, 2005. 8th International Conference on Management of Multimedia Networks and Services (MMNS 2005).

[3] H. Gascon, D. Diez, J. Garcia, F. Valera, C. Guerrero and A. Azcorra. Designing a broadband residential gateway using click! modular router. In IFIP EUNICE (Networked applications), Jul 2005. Madrid (Spain).

[4] M. Handley and V. Jacobson. SDP: Session Description Protocol. RFC 2327 (Proposed Standard), Apr. 1998. Updated by RFC 3266.

[5] ITU-T NGN Working Definition. http://www.itu.int/ITUT/studygroups/com13/ngn2004/working_definition.html [31-08-2006].

[6] E. Kohler, R. Morris, B. Chen, J. Jannotti, and M. F. Kaashoek. The Click Modular Router Project. Internet, May 2006. http://www.read.cs.ucla.edu/click/.

[7] MUSE. Multi Service Access Everywhere. Internet, May 2006. http://www.ist-muse.org/.

[8] J. Rosenberg and H. Schulzrinne. An Offer/Answer Model with Session Description Protocol (SDP). RFC 3264 (Proposed Standard), June 2002.

[9] J. Rosenberg, H. Schulzrinne, G. Camarillo, A. Johnston, J. Peterson, R. Sparks, M. Handley, and E. Schooler. SIP: Session Initiation Protocol. RFC 3261 (Proposed Standard), June 2002. Updated by RFCs 3265, 3853, 4320.

[10] TISPAN. ETSI ES 282001 V1.1.1: "Telecommunications and Internet converged Services and Protocols for Advanced Networking (TISPAN); NGN Functional Architecture Release 1", August 2005.

[11] TISPAN. ETSI ES 282003 V1.1.1: "Telecommunications and Internet converged Services and Protocols for Advanced Networking (TISPAN); Resource and Admission Control Sub-system (RACS); Functional Architecture.", March 2006.

[12] TISPAN. ETSI ES 282004 v1.1.1: "Telecommunications and Internet converged Services and Protocols for Advanced Networking (TISPAN); NGN Functional Architecture; Network Attachment Sub-System (NASS).”, June 2006.
[13] TISPAN. ETSI ES 282007 V1.1.1: "Telecommunications and Internet converged Services and Protocols for Advanced Networking (TISPAN); IP Multimedia Subsystem (IMS); Functional Architecture.", June 2006.

[14] TISPAN. ETSI ES 283 026 V1.1.1: "Telecommunications and Internet converged Services and Protocols for Advanced Networking (TISPAN); Resource and Admission Control; Protocol for QoS reservation information exchange between the Service Policy Decision Function (SPDF) and the Access-Resource and admission Control Function (ARACF) in the Resource and Protocol specification", April 2006.

[15] TISPAN. ETSI TR 180001 V1.1.1: "Telecommunications and Internet converged Services and Protocols for Advanced Networking (TISPAN); NGN Release 1; Release definition.”, March 2006.

[16] TISPAN. ETSI TS 183017 V1.1.1: "Telecommunications and Internet converged Services and Protocols for Advanced Networking (TISPAN); Resource and Admission Control; DIAMETER protocol for session based policy set-up information exchange between the Application Function (AF) and the Service Policy Decision Function (SPDF): Protocol specification.”, March 2006. 